\title{
Crystal structure of the kinesin motor domain reveals a structural similarity to myosin
}

\author{
F. Jon Kull", Elena P. Sablin ${ }^{*}$, Rebecca Lau ${ }^{*}$, Robert J. Fletterick ${ }^{*}, \dagger$, and Ronald D. Vale ${ }^{*}, \dagger, \ddagger$ \\ * Department of Biochemistry/Biophysics, University of California, San Francisco, California \\ 94143, USA \\ † Department of Pharmacology, University of California, San Francisco, California 94143, USA \\ ‡ Howard Hughes Medical Institute, University of California, San Francisco, California 94143, \\ USA
}

\begin{abstract}
Kinesin is the founding member of a superfamily of microtubule-based motor proteins that perform force-generating tasks such as organelle transport and chromosome segregation ${ }^{1,2}$. It has two identical 960 -amino-acid chains containing an amino-terminal globular motor domain, a central $\alpha$-helical region that enables dimer formation through a coiled-coil, and a carboxy-terminal tail domain that binds light chains and possibly an organelle receptor ${ }^{1}$. The kinesin motor domain of $\sim 340$ amino acids, which can produce movement in vitro ${ }^{3}$, is much smaller than that of myosin ( $~ 850$ amino acids) and dynein (1,000 amino acids), and is the smallest known molecular motor. Here, we report the crystal structure of the human kinesin motor domain with bound ADP determined to 1.8 - $\AA$ resolution by $\mathrm{X}$-ray crystallography. The motor consists primarily of a single $\alpha / \beta$ arrowhead-shaped domain with dimensions of $70 \times 45 \times 45 \AA$. Unexpectedly, it has a striking structural similarity to the core of the catalytic domain of the actin-based motor myosin. Although kinesin and myosin have virtually no amino-acid sequence identity, and exhibit distinct enzymatic ${ }^{4-} 6$ and motile $7^{-} 10$ properties, our results suggest that these two classes of mechanochemical enzymes evolved from a common ancestor and share a similar force-generating strategy.
\end{abstract}

The structure of the kinesin motor domain (amino acids 1-349) complexed with MgADP was determined by X-ray crystallography using multiple isomorphous derivatives (Table 1; Fig. 1a). The fragment of the kinesin motor visible in our structure (amino acids 7-325) is composed of a core eight-stranded, mostly parallel $\beta$-sheet that is flanked on each side by three $\alpha$-helices and a small lobe composed of a three-stranded $\beta$-sheet (see Fig. 1 legend for structure description). The MgADP is bound in a rather open and solvent-exposed cleft located in the upper notch of the arrowhead (Fig. 1b).

Although kinesin contains a phosphate-binding loop (P-loop) (amino acids 84-92) that is identical in structure to the P-loops of adenylate kinase, transducin, Ras and $\operatorname{Rec} \mathrm{A}^{11}$, other secondary structural elements of kinesin superimpose poorly with these proteins. In contrast, secondary structural elements of kinesin and myosin were discovered to overlap with one another in a striking manner in the area of the catalytic domain surrounding the nucleotide (Fig. $2 a, b$ ). Remarkably, seven of the eight core $\beta$-strands (the interrupted edge strand, $\beta 5$, being the exception) and all six major helices of the kinesin motor domain overlap with

Correspondence to: Ronald D. Vale.

Coordinates referred to in this paper will be deposited in the Brookhaven Protein Database within a year of publication. 
corresponding structural elements in myosin. The r.m.s. deviation of 183 amino-acid $\alpha$ carbon positions in the overlapping regions is $3.5 \AA$. This result was quite unexpected, given the much greater size of the myosin motor domain and the lack of sequence similarity between the two proteins. A computer alignment of tertiary structures ${ }^{12}$ that detected the significant similarity between kinesins and myosins did not detect a similarity between kinesin and any other known protein structure (L. Holm, personal communication).

The structural similarity between kinesin and myosin is not composed of contiguous sequence, being interrupted by large insertions of amino acids (Fig. 2c). Of the core structural elements, six of the seven $\beta$-strands (the edge strand $\beta 2$ being the exception) and all six $\alpha$-helices run in the same direction. Although the position and direction of these strands and helices are similar, two secondary structural elements are in a different relative location in the primary sequence. These different topological connections can be explained by a few distinct genetic insertions to a common ancestor of the kinesin and myosin families (F.J.K. et al., manuscript in preparation). As well as having longer $\mathrm{N}$ - and $\mathrm{C}$-terminal extensions from the core, myosin has two larger insertions compared to kinesin: residues 270-450 (insertion 1), which correspond to a smaller insertion in kinesin (amino acids 138173); and residues 506-649 (insertion 2), which correspond to loop L12 in kinesin (amino acids 272-280). (Unless indicated, residue numbers refer to chicken skeletal muscle myosin.)

To produce movement, a motor must hydrolyse nucleotide and harness stored chemical energy to generate force while bound to a polymer. The unexpected structural similarity of kinesins and myosins provides an opportunity to identify common features in the mechanisms of motor function. As described below, comparison of the nucleotide-binding region of these two motors shows how conformational changes in this environment can be transmitted and amplified into mechanical work.

The nucleotide-binding cleft is much more open in kinesin than in myosin, even though the rate constant of ADP dissociation from kinesin $\left(\sim 0.005 \mathrm{~s}^{-1}\right)^{4,6}$ is much slower than from myosin $\left(1 \mathrm{~s}^{-1}\right)^{13}$. In the kinesin pocket, the adenine is sandwiched by a stacking interaction with His 93 from below and by hydrophobic interactions with Pro 17 and the methylene group of Arg 16 from above (Fig. 3a). The last two residues are positioned similarly to Asn 127 and Pro 128 in myosin. The adenine also binds to water molecules coordinated to Pro 17 and Thr 94. The ribose makes no direct protein contacts. Below the adenine, kinesin features a prominent loop (L5; amino acids 96-106) that disrupts the P-loop helix ( $\alpha 2)$, which does not have a counterpart in myosin. Although this loop is found in all members of the kinesin superfamily, L5 does not contain a hydrophobic core and has no direct contact with the nucleotide.

Kinesin lacks certain loops that are present in myosin's nucleotide-binding cleft (Fig. 3a). Two unique myosin loops between amino acids 320-327 and 127-136 cover the nucleotidebinding region and position Ser 324 and Trp 131 within crosslinking distance of the adenine14. A third unique loop (amino acids 233-246, with Arg 239 at its apex), together with the P-loop, creates a narrow tunnel between the ribose and the $\gamma$-phosphate region ${ }^{15}$. The corresponding loop (L9; amino acids 190-204) in the kinesin structure contains fewer residues and is positioned further away from the $\alpha, \beta$ phosphates (Fig. $3 a, b$ ). The different loop positions in the two motors may explain why phosphate release following hydrolysis is slow $\left(0.1 \mathrm{~s}^{-1}\right)$ and rate limiting in the myosin ATPase cycle ${ }^{16}$, but occurs at much faster rates $\left(>13 \mathrm{~s}^{-1}\right)$ in the case of kinesin ${ }^{5,6}$.

Although several loops near the entrance of the nucleotide pocket are different in kinesin and myosin, comparison of the $\gamma$-phosphate environment of the nucleotide pockets reveals 
intriguing similarities (Fig. 3b). Both kinesin (amino acids 201-203) and myosin (243-245) contain the universally conserved motif of Ser-Ser-Arg, which occupies a similar position in space. Crosslinking studies with ADP-vanadate ${ }^{17}$ and the atomic structure of Dictyostelium myosin with bound $\mathrm{MgADP}-\mathrm{AlF}_{4}$ (ref. 15), which is thought to be a MgADP-Pi analogue, indicate that the second member of the triplet, Ser 243, can interact with the $\gamma$-phosphate. By analogy, Ser 202 in kinesin, which is only $7 \AA$ away from the $\beta$-phosphate, may also interact with the $\gamma$-phosphate when ATP is bound in the active site. A second region of similarity is seen in the loop extending from the adjacent $\beta$-strand ( $\beta 7)$, where the conserved kinesin sequence Asp-Leu-Ala-Gly-Ser-Glu (amino acids 231-236) covers the rear of the nucleotide cleft in a similar position to a nearly identical sequence in myosin (Asp-Ile-Ala-Gly-PheGlu; 463-468) (Fig. 3b). The amide group of Gly 466 in this segment of myosin moves to form a hydrogen bond with the AlF4-ADP complex, which results in the formation of a new salt bridge between Glu 468 and Arg 245 (ref. 15). These rearrangements have been implicated in transmitting conformational changes from the nucleotide cleft of the actinbinding site15. Because of its similar location, Gly 234 in kinesin may serve a similar function to Gly 466 in myosin (see also ref. 18). Moreover, we speculate that movement of this glycine towards the nucleotide in the ATP state could bring loops L9 and L11 together, thereby disrupting the Arg 203-Glu 199 charge pair and allowing Glu 236 and Arg 203 to form a salt bridge, as described for myosin.

Although the residues of kinesin that interact with microtubules have not been identified by biochemical studies, comparison of the kinesin structure with myosin suggests a possible location for a polymer binding site. The actin-binding interface of myosin is distributed primarily over three regions: a loop from amino acids 405-415, which is part of insertion 1 (Fig. 2c), as well as a helical segment (529-558) and a disordered loop (626-647) that belong to insertion 2 . The kinesin analogue of myosin insertion 1 is a smaller but wellordered structure that extends from $\beta 4$, and includes the $\beta 5 \mathrm{a}$ and $\beta 5 \mathrm{~b}$ strands and loop L8 (amino acids 138-173). The kinesin analogue of myosin insertion 2 is a short, extended loop (L12, amino acids 272-280) that contains highly conserved hydrophobic residues (Fig. 3c). Because these kinesin elements extend from the motor core in the same locations as the actin-binding insertions in myosins, they represent candidate regions for mediating interactions with microtubules.

How might the kinesin motor amplify small sub-nanometre conformational changes in the nucleotide pocket into larger translational movements? The side of the kinesin motor opposite the nucleotide pocket forms a network of structural elements that could transduce conformational changes to the putative micro-tubule-binding region and to the $\mathrm{C}$ terminus of the motor domain, which is connected to the long stalk (Fig. 3c). This network includes the putative microtubule-binding site described above (L12; amino acids 272-280), the side chains of which form interactions with both $\alpha 4$ and $\alpha 5$. Conformational changes in the filament-binding site could also affect $\alpha 6$, which is the C-terminal helix visible in the structure. A similar network is seen in myosin, where two helices corresponding to $\alpha 4$ and $\alpha 5$ in kinesin flank the large actin-binding region insert (amino acids 507-648). The myosin equivalent of $\alpha 4$ is thought to be particularly important in the conformational change mechanism, as it contains highly conserved residues and undergoes rotational movements in different nucleotide states ${ }^{15}$. After the actin-binding insertion, myosin then follows a similar path to kinesin through a core $\beta$-strand (amino acids 668-675; equivalent to $\beta 8$ in kinesin) and into a helix (690-697; equivalent to $\alpha 6$ helix). The C-terminal region of the kinesin structure corresponds to an area of considerable interest in myosin (the reactive thiol region) that is thought to propagate and amplify nucleotide-dependent conformational changes 15.

The amplifying structure in myosin is probably an 85 - $\AA \alpha$-helix that extends from the catalytic core and is stabilized by two associated light chains. This helix can move by $35 \AA$ 
or more in different nucleotide states19,20. This 'lever arm' action is thought to constitute the myosin power stroke that underlies force generation. Although there is no comparable element visible in kinesin, circular dichroism spectral analysis of bacterially expressed kinesin and synthetic peptides suggest that residues within amino acids 330-370 (the neck region) form a long helix that forms a coiled-coil (R. Hodges, personal communication; T. Shimizu and H. Morii, personal communication). If this helix were stable enough to form a rigid 'lever arm', and undergoes a $\sim 30^{\circ}$ rotation as observed in myosin ${ }^{20}$, the translation produced would be $\sim 25 \AA$, much shorter than the $80 \AA$ necessary to move from one tubulin binding site to the next during each ATPase cycle9'10. However, movement of this putative amplifier in the native kinesin dimer could communicate mechanical strain to the partner head, biasing it to attach to a forward binding site on the microtubule21. This could be accomplished by relatively small conformational changes in the leading motor head that could be communicated to the partner head through a linkage between the two polypeptide chains. This cooperative mechanism could also explain how kinesin moves for long distances along a microtubule without dissociating ${ }^{7,8,22,23}$, whereas myosin spends most of its ATPase cycle dissociated from $\operatorname{actin}^{24}$.

In conclusion, the considerable structural similarities between kinesin and the core of myosin suggest that these two motor proteins may adopt a similar strategy for converting chemical energy into mechanical work. Kinetic differences in the ATPase cycles may result from interactions of the nucleotide with loops that are unique for each motor. However, our data show that residues and structural elements mediating conformation changes between ATP and ADP states may be similar in the myosin and kinesin superfamilies (see also ref. 18). Moreover, amplification of conformational changes may occur in the C-terminal region just beyond the core of both motor proteins. Adaptations in this amplifier region may enable different types of motility between kinesin and myosin as well as between motor proteins within a superfamily.

\section{Acknowledgments}

We thank N. Naber for preparation of 2'-I-ATP, C. Coppin for discussing models of kinesin movement, and P. Focia, P. Foster, L. Brinen, T. Stout, A. McDonald and F. Malik for help. This work was supported by NIH Program Project grants.

\section{References}

1. Bloom, G.; Endow, S. Motor Proteins 1: Kinesin. Academic; London: 1994.

2. Goldstein LS. A Rev Genet 1993;27:319-351.

3. Yang JT, Saxton WM, Stewart RJ, Raff EC, Goldstein LS. Science 1990;249:42-47. [PubMed: 2142332]

4. Hackney DD. Proc natn Acad Sci USA 1988;85:6314-6318.

5. Gilbert SP, Webb MR, Brune M, Johnson KA. Nature 1995;373:671-676. [PubMed: 7854446]

6. Ma YZ, Taylor EW. Biochemistry 1995;34:13233-13241. [PubMed: 7548087]

7. Howard J, Hudspeth AJ, Vale RD. Nature 1989;342:154-158. [PubMed: 2530455]

8. Block SM, Goldstein LS, Schnapp BJ. Nature 1990;348:348-352. [PubMed: 2174512]

9. Svoboda K, Schmidt CF, Schnapp BJ, Block SM. Nature 1993;365:721-727. [PubMed: 8413650]

10. Coppin CM, Finer JT, Spudich JA, Vale RD. Proc natn Acad Sci USA. (in the press).

11. Story RM, Steitz TA. Nature 1992;355:374-376. [PubMed: 1731253]

12. Holm L, Sander C. J molec Biol 1993;233:123-138. [PubMed: 8377180]

13. Bagshaw CR, Trentham DR. Biochem J 1974;141:331-349. [PubMed: 4281653]

14. Grammar JC, Kuwayama H, Yount RG. Biochemistry 1993;32:5752-5732. [PubMed: 8504093]

15. Fisher AJ, et al. Biochemistry 1995;34:8960-8972. [PubMed: 7619795] 
16. Taylor, EW. The Heart and Cardiovascular System. Fozzard, HA., editor. Raven; New York: 1992. p. 1281-1293.

17. Yount RG, Cremo CR, Grammar JC, Kerwin BA. Phil Trans R Soc Lond B 1992;336:55-61. [PubMed: 1351297]

18. Sablin EP, Kull FJ, Cooke R, Vale RD, Fletterick RJ. Nature 1996;380:555-559. [PubMed: 8606780]

19. Whittaker M, et al. Nature 1996;378:748-751. [PubMed: 7501026]

20. Jontes JD, Wilson-Kubalek EM, Milligan RA. Nature 1995;378:751-753. [PubMed: 7501027]

21. Peskin CS, Oster G. Biophys J 1995;68:202s-211s. [PubMed: 7787069]

22. Vale RD, et al. Nature 1996;380:451-453. [PubMed: 8602245]

23. Berliner E, Young EC, Anderson K, Mahtani HK, Gelles J. Nature 1995;373:718-721. [PubMed: 7854458]

24. Spudich JA. Nature 1994;372:515-518. [PubMed: 7990922]

25. Navone F, et al. J Cell Biol 1992;117:1263-1275. [PubMed: 1607388]

26. Fujiwara S, Kull FJ, Sablin EP, Stone DB, Mendelson RA. Biophys J 1995;69:1563-1568. [PubMed: 8534827]

27. Naber N, Matuska M, Sablin EP, Pate E, Cooke R. Protein Sci 1995;4:1824-1831. [PubMed: 8528080]

28. McRee, DE. Practical Protein Crystallography. Academic; San Deigo: 1993.

29. Collaborative Computing Project No. 4. Acta crystallogr 1994;D50:760-763.

30. Brunger, AT. X-PLOR Version 3.1. A system for X-ray Crystallography and NMR. Yale Univ. Press; New Haven, CT: 1992.

31. Laskowski RA, MacArthur MW, Moss DS, Thornton JM. J appl Crystallogr 1993;26:283-291.

32. Kraulis PJ. J appl Crystallogr 1991;24:946-950.

33. Rayment I, et al. Science 1993;261:58-65. [PubMed: 8316858]

34. Wells JA, Yount RG. Proc natn Acad Sci USA 1979;76:4966-4970. 

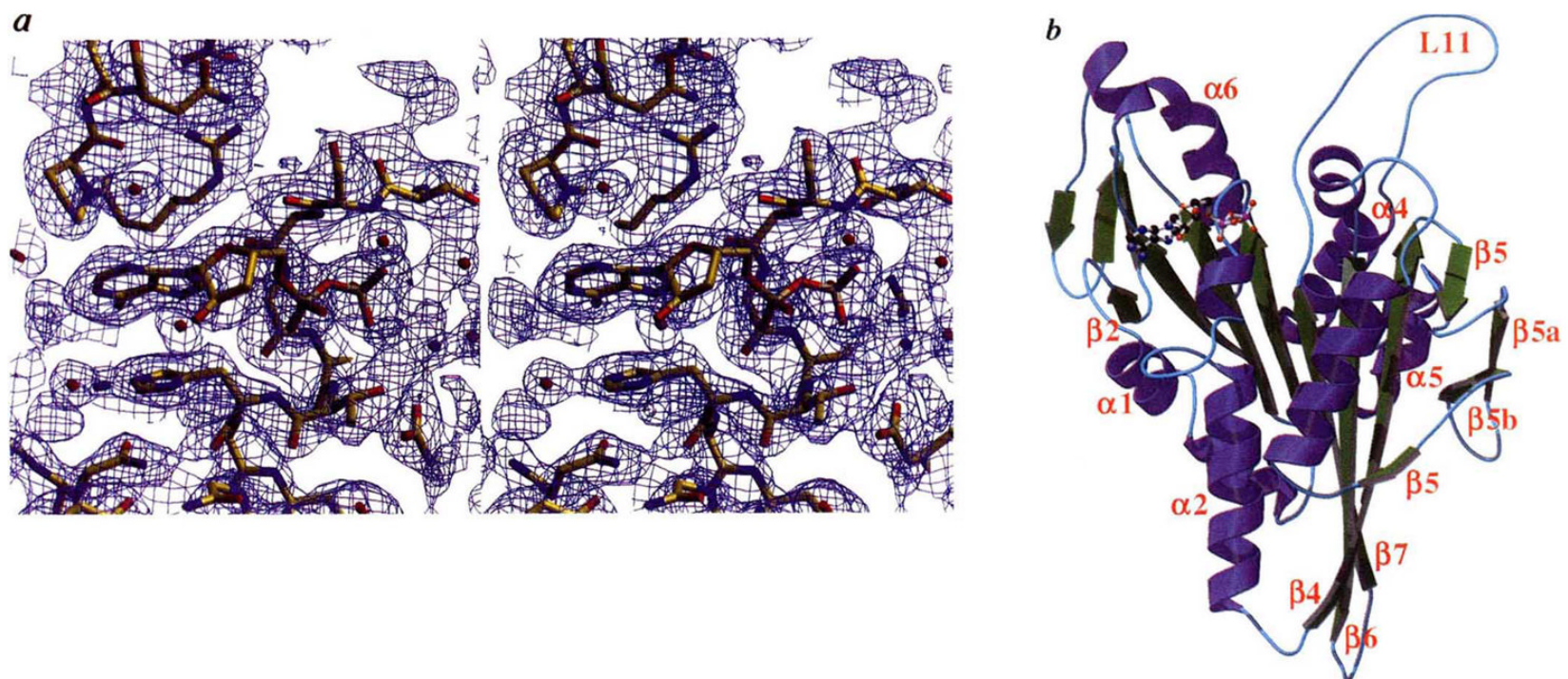

FIG. 1.

Structure of the human kinesin motor domain. $a$, Stereo view showing electron density in the MgADP binding area from the final SIGMAA weighted 1.8- $\AA 2 F_{\mathrm{O}}-F_{\mathrm{c}}$ map. The final refined structure is shown. The red spheres indicate water molecules and the purple sphere indicates the magnesium ion. Map contours are a $1 \sigma . b$, MOLSCRIPT ${ }^{32}$ rendition of the kinesin structure, in which $\beta$-strands are shown in green, $\alpha$-helices in purple, and loops in cyan. The ADP is shown as a ball-and-stick figure. The small lobe containing a threestranded $\beta$-sheet is seen in the upper left of the molecule. Two adjacent strands of the core $\beta$ sheet, $\beta 4$ (amino acids 126-138) and $\beta 6$ (206-216), are unusually long and run the entire length of the molecule; one of the edge strands ( $\beta 5 ; 141-144$ and $171-173)$ contains an insertion of 24 amino acids that forms an extended loop with two short $\beta$-strands that are isolated from the main $\beta$-sheet. Several of the $\alpha$-helices also exhibit unusual features. Most unusual is $\alpha 2$ (91-122), which contains a 9-amino-acid insertion (97-104) flanked by two conserved glycines. This 'hairpin' loop extends perpendicular to the helix, but leaves the overall helical path of $\alpha 2$ unperturbed. Helices $\alpha 4$ (257-269) and $\alpha 5$ (281-292) are notable in that they are oriented at $45^{\circ}$ to the $\beta$-sheet, unlike the other four helices that are in a parallel orientation. These helices are the only ones linked directly by a short loop rather than by an intervening $\beta$-strand. Although loop L11 is shown, it is disordered at the tip (238-254) and is not part of the present model. 

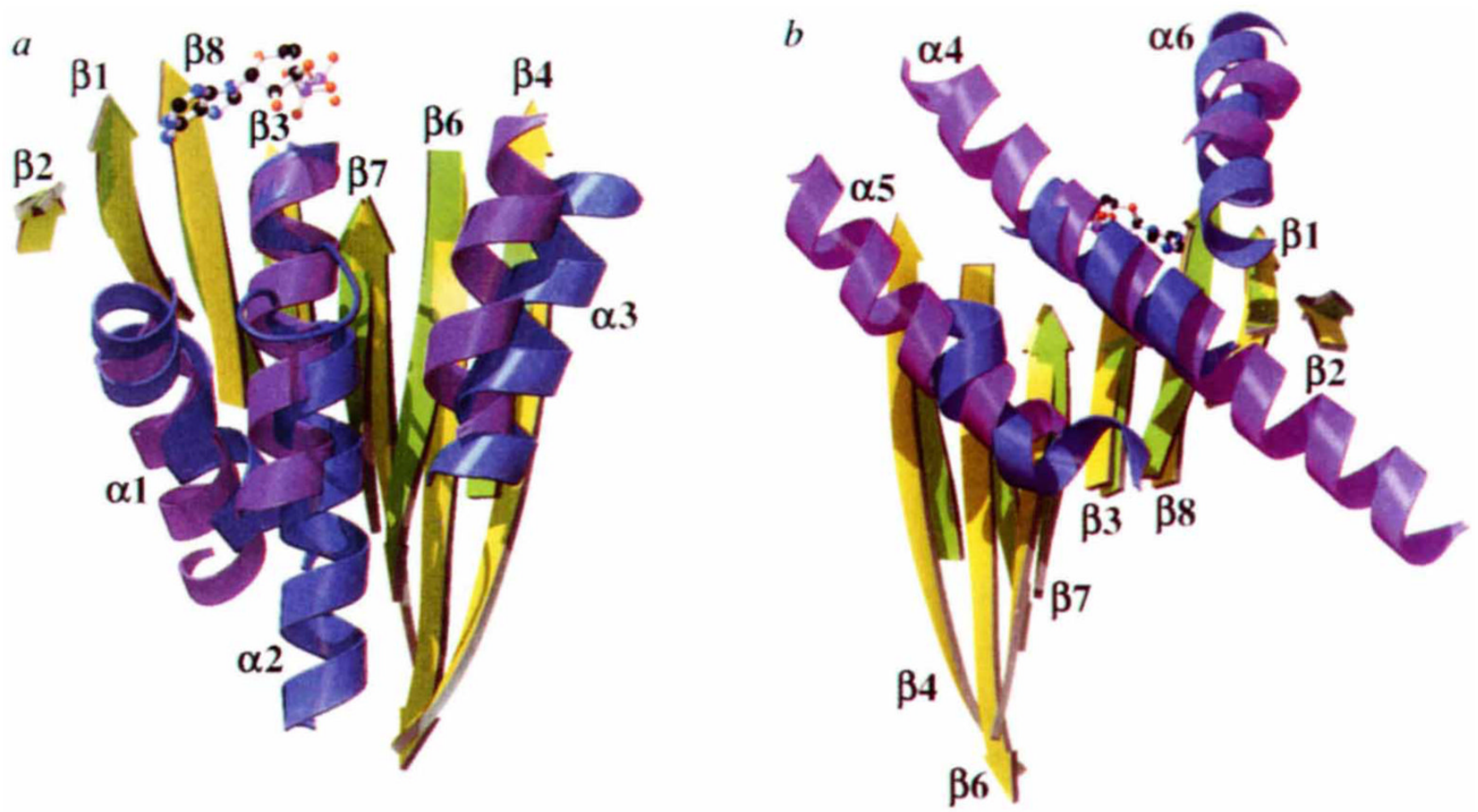

c

\section{Myosin}

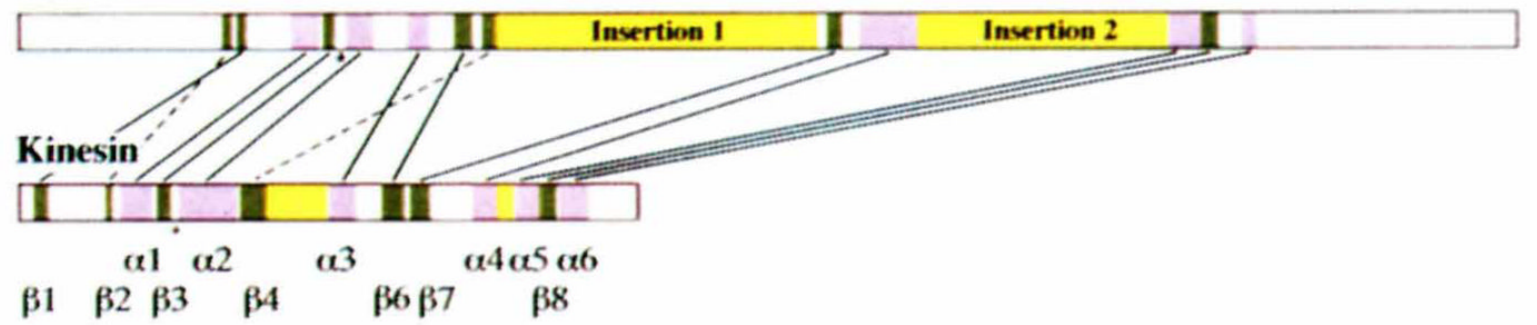

Amino Acid Number

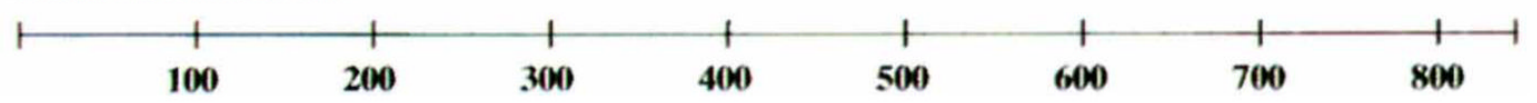

FIG. 2.

Comparison of overlapping secondary structure in kinesin and myosin. The overlap was achieved by aligning nine $\alpha$-carbon atoms (kinesin amino acids $84-92$ and myosin 178-186) in the P-loops of the two structures. The similarity between kinesins and myosins was first noted when the ncd tertiary structure 18 was aligned with other proteins in the coordinate data bank12 (L. Holm, personal communication). $a$, Front view of the molecule, showing overlapping $\beta$-sheets and the three front $\alpha$-helices. Secondary structure elements of kinesin are indicated. Myosin residue numbers refer to chicken skeletal muscle myosin. Shown are: $\beta$-strands (kinesin (k) yellow; myosin (m), green); left to right, $\beta 2, \mathrm{k} 50-\mathrm{k} 52, \mathrm{~m} 116-119 ; \beta 1$, k9-k15, m122-m126; $\beta 8$, k295-k302, m668-m675; $\beta 3$, k79-k84, m173-m177; $\beta 7$, k222k231, m457-m463; $\beta 6, \mathrm{k} 206-\mathrm{k} 216, \mathrm{~m} 247-\mathrm{m} 255 ; \beta 4, \mathrm{k} 126-\mathrm{k} 138, \mathrm{~m} 263-\mathrm{m} 268$. Helices 
(kinesin, blue; myosin, purple); left to right, $\alpha 1$, k58-k74, m155-m169; $\alpha 2$, k91-k122, m186-m199; $\alpha 3, \mathrm{k} 176-\mathrm{k} 189, \mathrm{~m} 220-\mathrm{m} 231 . b$, Rear view of the molecule, showing overlapping $\beta$-sheets and the three rear $\alpha$-helices. $\beta$-strands, same as in $a$, but rotated by $180^{\circ} ; \alpha$-helices, left to right, $\alpha 5, \mathrm{k} 281-\mathrm{k} 292$, m649-m665; $\alpha 4, \mathrm{k} 257-\mathrm{k} 269$, m475-m506; $\alpha 6$, k306-k320, m690-m697. $c$, Placement of corresponding structural elements in the linear sequence of kinesin and myosin. Helices and strands are indicated in purple and green, respectively. Insertions in the core motor domains are shown in yellow. In myosin, these insertions contain elements that interact with $\operatorname{actin}^{33}$. Lines connect overlapping structural elements; broken lines indicate structural elements that are in a different relative location in the primary sequence; the asterisk indicates the position of the P-loops. 

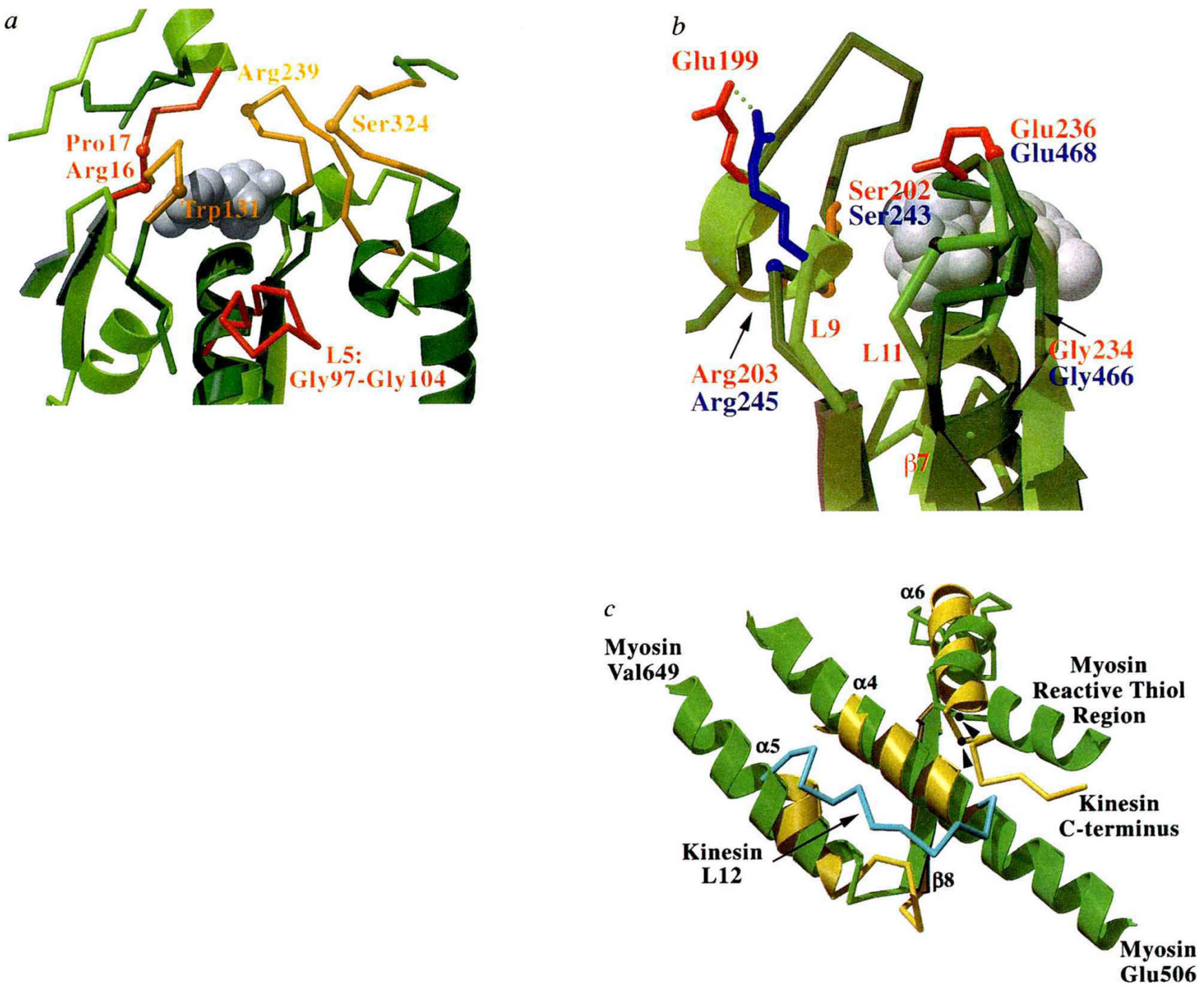

FIG. 3.

Nucleotide environment of kinesin and comparison of functional regions with myosin, $a$, Loops surrounding the entrance to the nucleotide-binding pocket are shown in this view of kinesin (light green with red loops) overlaid with myosin (dark green with orange loops). The bound ADP is shown as grey spheres, and highlighted residues and loops are discussed in the text. $b$, The back of the nucleotide-binding pocket with kinesin in light green and myosin in dark green. Residues that may be involved in $\gamma$-phosphate sensing are indicated (kinesin residues labelled in red, myosin in blue). Side chains are coloured red for Glu, blue for Arg, orange for Ser, and grey for Gly. Myosin residues are indicated by their $\alpha$-carbon positions. The bound ADP is shown as grey spheres. This view has been rotated by approximately $180^{\circ}$ relative to that in $a$. c , Structures potentially involved in transducing conformational changes from the nucleotide and filament binding site to a mechanical amplifier in kinesin (yellow) and myosin (green). Secondary structure elements of kinesin are indicated. Orientation is similar to that in Fig. 2b. The kinesin loop (L12; amino acids 272-280) that may be involved in microtubule binding is shown in light blue. The 142amino-acid actin-binding domain of myosin is not shown, but is located between myosin residues Glu 506 and Val 649. Myosin's reactive thiol region that may bend or melt during 
myosin's ATPase cycle ${ }^{34}$ and the $\mathrm{C}$ terminus of the current kinesin model are indicated. The position of glycine residues that may be involved as pivot points for conformational movements are shown for myosin Gly 699 and kinesin Gly 319 (arrowheads). 


\section{TABLE 1}

Summary of data collection, phase calculation, model building and refinement

\begin{tabular}{|c|c|c|c|}
\hline & Native & I-ATP & EMTS \\
\hline \multicolumn{4}{|l|}{ Cell dimensions: } \\
\hline$a(\AA)$ & 48.54 & 48.66 & 48.68 \\
\hline$b(\AA)$ & 67.94 & 67.88 & 67.73 \\
\hline$c(\AA)$ & 112.95 & 113.37 & 112.76 \\
\hline \multicolumn{4}{|l|}{ Data collection: } \\
\hline Resolution $(\AA)$ & 1.8 & $2.5^{*}$ & 2.5 \\
\hline$R_{\mathrm{sym}}(\%)^{\dagger}$ & 6.1 & 17.2 & 14.1 \\
\hline Observed reflections & 101,090 & 40,935 & 96,338 \\
\hline Unique reflections & 30,582 & 11,951 & 12,726 \\
\hline Completeness (\%) & 82.4 & 87.4 & 91.1 \\
\hline \multicolumn{4}{|l|}{ Phasing (15.0-3.0 ̊̊): } \\
\hline$R_{\text {scal }}{ }^{*}$ & - & 14.2 & 21.2 \\
\hline Number of sites & - & 1 & 3 \\
\hline \multirow[t]{3}{*}{ Relative occupancy } & - & - & 1.0 \\
\hline & & & 0.63 \\
\hline & & & 0.40 \\
\hline Phasing power ${ }^{\S}$ & & 0.84 & 1.95 \\
\hline$R_{\text {Cullis }} \|$ & & 0.76 & 0.59 \\
\hline Mean figure of merit & 0.48 & & \\
\hline \multicolumn{4}{|l|}{ Refinement (6.0-1.8̊): } \\
\hline$R_{\text {cryst }}(\%)^{I l}$ & 23.4 & & \\
\hline$R_{\text {free }}(\%)^{\#}$ & 30.7 & & \\
\hline Average $B$-factor $\left(\AA^{2}\right)$ & 26.1 & & \\
\hline
\end{tabular}

The first 349 amino acids of the human kinesin gene 25 containing the conserved motor domain were expressed in bacteria and purified as described $^{26}$. Crystals were obtained using sitting drops containing $20 \mu 1$ of $\sim 5 \mathrm{mgml}^{-1}$ protein in $50 \mathrm{mM} \mathrm{Na}$ Acetate, $\mathrm{pH} 4.6,75 \mathrm{mM} \mathrm{KCl}, 3.5 \%$ $(\mathrm{w} / \mathrm{v})$ PEG 4000, $2.5 \mathrm{mM}$ ATP, $10 \mathrm{mM} \mathrm{MgCl} 2$. Crystals $(1,000 \times 150 \times 40 \mu \mathrm{m})$ appeared after 2-3 days at $4{ }^{\circ} \mathrm{C}$, and were of the orthorhombic space group $P 212121$ with one molecule with bound MgADP in the asymmetric unit. All data collection was performed at $-170{ }^{\circ} \mathrm{C}$ using a $30 \%$ (v/v) solution of glycerol/precipitant solution as a cryosolvent. The native data were collected at Stanford Synchrotron Laboratory Radiation beamline $7-1(\lambda=1.08 \AA)$, and derivative data were collected using a 300-mA, 50-kV rotating anode X-ray source. The first derivative was obtained by co-crystallizing K349 with $2.5 \mathrm{mM} 2$ '-iodo-ATP (I-ATP) 27 instead of ATP. The second derivative was obtained by soaking native crystals in the presence of $0.1 \mathrm{mM}$ ethyl mercurothiosalycilate (EMTS) for $\sim 2 \mathrm{~h}$. The programs DENZO and SCALEPACK (Z. Otwinowski and W. Minor) were used to index and integrate all data sets. Heavy-atom sites were initially found by analysis of difference Patterson maps and refined using the program XTALVIEW28. One I-ATP derivative site and three Hg sites (bound to Cys 174, Cys 168 and Cys 294) were found for each kinesin molecule. Heavy-atom positions were further refined, and multiple isomorphous replacement (MIR) phases were calculated using the program MLPHARE, and the initial 3.0-Å MIR map and density-modified map (solvent flattening/histogram matching using the program DM) were calculated using the CCP4 suite of programs29. The K349 chain could be traced using these maps in combination with density skeletons created using the program 0 (T. A. Jones and M. Kjeldgaard). The quality of initial maps was better than expected from the phasing statistics, as indicated by visible carbonyl density in several helices. After a chain of $\sim 160$ alanines and 40 residues was placed, the chain was compared with the working model of the NCD structure. Comparison of the two independently derived chains confirmed that the chains were correctly placed and connected. An initial, four-fragment model of a $~ 275$-amino-acid fragment of the kinesin motor was then refined in the program X-PLOR30 using a least-squares protocol and, later, least-squares and simulated annealing protocols. Individual $B$-factors were refined isotropically. The structure satisfied the PROCHECK ${ }^{31}$ criteria at $1.8 \AA$. The final structure contains amino acids $7-325, \mathrm{MgADP}$ and 30 water molecules. 
* I-ATP data were processed to $2.5 \AA$; diffraction was weak beyond $3.0 \AA$.

${ }^{\dagger} R_{\mathrm{Sym}}=100 \times \Sigma_{\mathrm{hkl}} \Sigma_{\mathrm{i}}\left|I_{\mathrm{i}}-I\right| / \Sigma_{\mathrm{hkl}} \Sigma_{\mathrm{i}} \mathrm{I}_{\mathrm{i}}$. All positive, non-zero reflections $\left(\mathrm{O}_{\sigma}\right.$ intensity cut-off $)$ were included in scaling and merging.

${ }^{*} R_{\text {Scale }}=100 \times \Sigma_{\mathrm{hkl}}\left|F_{\mathrm{PH}}-F_{\mathrm{H}}\right| / \Sigma_{\mathrm{hkl}}\left|F_{\mathrm{P}}\right|$. All positive, non-zero reflections were included in this calculation.

$\S_{\text {Phasing power }}=\left\langle F_{\mathrm{H}}\right\rangle /\langle E\rangle$, where $\left\langle F_{\mathrm{H}}\right\rangle$ is the mean calculated heavy-atom structure factor amplitude, and $\langle E\rangle$ is the mean estimated lack of closure. All positive, non-zero reflections were included in this calculation.

$\|_{R_{\text {cullis }}}=\langle E\rangle /\langle$ iso $\rangle$, where $\langle E\rangle$ is the mean estimated lack of closure and $\langle$ iso $\rangle$ is the isomorphous difference.

$I_{R_{\mathrm{cryst}}}=100 \times \Sigma_{\mathrm{hkl}}\left|F_{\mathrm{O}}-F_{\mathrm{C}}\right| / \Sigma_{\mathrm{hkl}}\left|F_{\mathrm{O}}\right|$, where $F_{\mathrm{O}}$ and $F_{\mathrm{C}}$ are observed and calculated structure factor amplitudes, respectively (for all data).

${ }^{\#} R_{\text {free }}$ was calculated using $10 \%$ of the data chosen randomly and omitted from the refinement. 